\title{
Les productions françaises d'oléagineux de spécialité : des démarches en filière pour créer de la valeur dans nos territoires
}

\author{
Perrine Tonin ${ }^{*}$ \\ Groupe AVRIL, Paris, France
}

Reçu le 1 mars 2018 - Accepté le 28 février 2018

\begin{abstract}
Résumé - Dans un contexte de demande de diversification de la part du consommateur, de recherche de nouveaux débouchés pour les producteurs et de mondialisation des marchés oléagineux, les acteurs de la filière française ont développé des productions de spécialité afin de redonner de la valeur ajoutée à la filière. Cette analyse dresse un panorama de ces productions à travers quatre exemples : la filière oléique pour des qualités nutritionnelles et fonctionnelles reconnues, la filière érucique pour une diversité de débouchés en oléochimie, la filière Fleur de Colza - démarche de long terme de partenariat agricole - et la filière bio - un marché jeune, dynamique, en cours de structuration. Dans chaque cas, la valorisation pour le producteur nécessite une démarche de filière avec l'accompagnement de l'ensemble des acteurs afin de valoriser l'origine française par la traçabilité, apporter conseil et formation aux agriculteurs, optimiser la supply chain, soutenir la recherche variétale et développer la contractualisation pluriannuelle.
\end{abstract}

Mots clés : oléagineux / spécialité / oléique / érucique / bio

\begin{abstract}
French oilseeds specialty productions: the "filière" approach to create value in our territories. Consumers want more diversification, producers are looking for new outlets for their productions, and oilseeds markets are now globalized. The French oilseeds sector's players have thus developed specialized productions in order to restore added value to the "filière" (this word has no equivalent in English. It describes all the economic players and activities coordinated all along the value chain). This review provides a comprehensive overview of these productions through four examples: the oleic sector has proven nutritional and functional values, the erucic sector opens opportunities in oleochemistry, the "Fleur de Colza" strengthens agricultural partnership on the long-term, and the organic sector is a new but dynamic and structuring market. In every case, the added value for the producer relies on a "filière" approach with the involvement of all stakeholders of the value chain in order to promote French origin with traceability, provide consulting and training for farmers, optimize the supply chain, support the varietal research, and develop multi-year contractualization.
\end{abstract}

Keywords: oilseeds / specialty / oleic / erucic / organic

\section{Introduction}

La filière oléoprotéagineuse française s'est développée depuis quarante ans afin de réduire sa dépendance aux protéines d'outre-Atlantique, suite à l'embargo américain sur les exportations de soja en 1973 en rassemblant tous les acteurs contribuant à la production, la commercialisation et la transformation des plantes riches en huiles et en protéines. Aujourd'hui, la surface domestique d'oléagineux s'est stabilisée autour de 2,2 Mha pour une production moyenne

*Correspondance : perrine.tonin@groupeavril.com de $7 \mathrm{Mt}$ depuis 10 ans (Agreste, 2017). La filière permet ainsi de répondre aux besoins en huiles et matières grasses dans l'alimentaire pour l'industrie agroalimentaire, la restauration hors domicile et la consommation d'huiles à domicile, de contribuer à la lutte contre le réchauffement climatique avec le biodiesel et de fournir une source de protéines françaises non OGM pour les élevages.

L'essentiel de ces productions entrent en compétition avec les autres productions mondiales telles que le canola canadien ou encore le tournesol ukrainien. En parallèle, les acteurs de la filière ont développé une diversité de productions d'oléagineux de spécialité, permettant de créer davantage de valeur ajoutée 
et de sécuriser des débouchés pour les producteurs. Les difficultés rencontrées pour développer les cultures de diversification listées par Valérie Duflot (Duflot, 2017) s'appliquent également dans le cas des spécialités oléagineuses : taille de marché limitant les investissements en sélection variétale, contraintes logistiques et industrielles supplémentaires, besoin d'information pour l'agriculteur, etc.

Cette analyse dresse un panorama de ces productions oléagineuses de spécialité et de leur structuration en filières, à travers quatre exemples: la filière oléique pour des qualités nutritionnelles et fonctionnelles reconnues, la filière érucique pour une diversité de débouchés en oléochimie, la filière Fleur de Colza - démarche de long terme de partenariat agricole- et la filière bio-un marché jeune, dynamique, en cours de structuration.

\section{La filière oléique pour des qualités nutritionnelles et fonctionnelles reconnues}

\subsection{Comparaison des huiles oléiques et principales huiles conventionnelles}

Le tournesol oléique contient en moyenne $84 \%$ d'acide oléique dans sa fraction lipidique, faisant de cette huile la plus riche en acide oléique, devant le colza oléique $(75 \%)$ ou encore le soja oléique (73\%). Par comparaison, le tournesol classique ne contient que $20 \%$ d'acide oléique (Fig. 1).

Ainsi, chacune des principales cultures oléagineuses existe dans sa version oléique : l'Europe cultive surtout du tournesol oléique, le Canada du canola oléique et les États-Unis du soja oléique.

\subsection{Le marché du tournesol oléique européen drivé par la demande alimentaire}

Si l'huile de tournesol classique représente $80 \%$ de la consommation européenne d'huile de tournesol, les $20 \%$ de consommation d'huile de tournesol oléique (HOSO) sont tirés par les demandes des industries agroalimentaires et de la restauration hors foyer. En effet, l'HOSO présente des atouts pour ces utilisateurs finaux : alternative aux acides gras trans, teneur plus faible en acides gras saturés, allongement de la durée de vie du produit, même stabilité aux températures élevées que l'huile de palme, goût neutre...

Le premier débouché de l'HOSO en Europe est le marché des snacks, avec $33 \%$ des débouchés (OSE Consulting, 2016), grâce au goût neutre et à la bonne stabilité de l'huile. Suit le marché des biscuits et produits de la boulangerie (18\%), pour lequel l'HOSO est une bonne alternative à l'huile de colza dans les pays d'Europe du Sud. La restauration professionnelle et l'industrie de la friture représentent respectivement $14 \%$ et $9 \%$ de la consommation d'HOSO. Pour ces marchés, la réduction des coûts bains de friture grâce à l'allongement de la durée des bains est déterminante.

\subsection{La France, un pionnier du tournesol oléique}

La production de tournesol oléique a commencé au début des années 2000 en Europe de l'Ouest, et particulièrement en France où des industriels et acteurs de la restauration ont lancé

\section{Profil d'acides gras des principals huiles végétales}
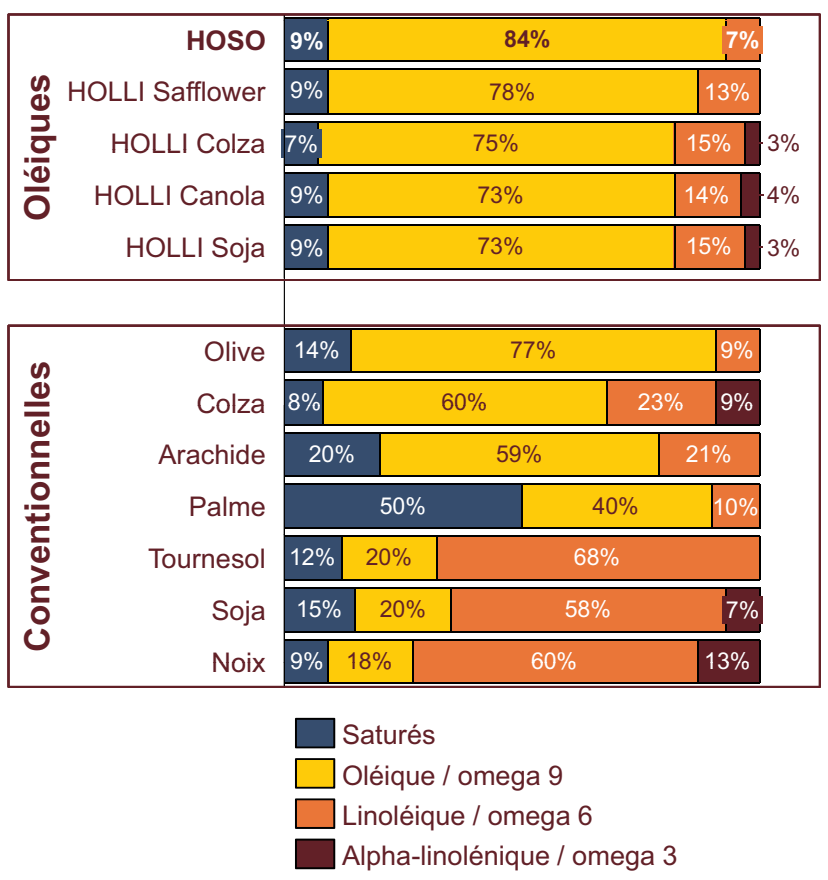

Fig. 1. Profil d'acides gras des principales huiles végétales. HOSO : «high oleic sunflower oil» (huile de tournesol oléique); HOLLI : «high oleic acid, low linolenic acid» (huile à forte teneur en acide oléique et faible teneur en acide linolénique).

Source: OSE Consulting.

les premiers partenariats pour tester l'HOSO en remplacement des huiles hydrogénées et de l'huile de palme.

Cette production initialement de niche est devenue un marché à forte valeur ajoutée en Europe de l'Ouest dès la fin des années 2000. La France s'est alors mise à produire plus de tournesol oléique que de tournesol classique, avec une part de l'oléique dans l'assolement qui oscille aujourd'hui entre $60 \%$ et $70 \%$ (Fig. 2). C'est à cette période que les principaux acteurs de l'industrie agroalimentaire et de la restauration ont opéré leur transition vers les huiles oléiques dans leurs recettes en Europe.

\subsection{Un marché qui doit relever le challenge de la montée en puissance d'autres pays}

Le tournesol oléique est donc une commodité au niveau français, mais reste une culture de niche au niveau mondial. Néanmoins, l'attractivité des marchés alimentaires européens a conduit la Russie et l'Ukraine à développer rapidement leur propre production. Entre 2011 et 2015, les surfaces de tournesol oléique en Russie plus Ukraine ont presque quadruplé: de 110 kha en 2011 à 420 kha en 2018 tandis que les surfaces européennes stagnaient entre 400 et $430 \mathrm{kha}$.

En conséquence, la production mondiale d'huile de tournesol oléique a fortement cru ces dernières années, engendrant un surplus d'offre par rapport à la demande sur le marché européen. Ce choc de production a eu pour effet d'annihiler la prime oléique - prime versée en plus du prix de la graine de tournesol classique. 


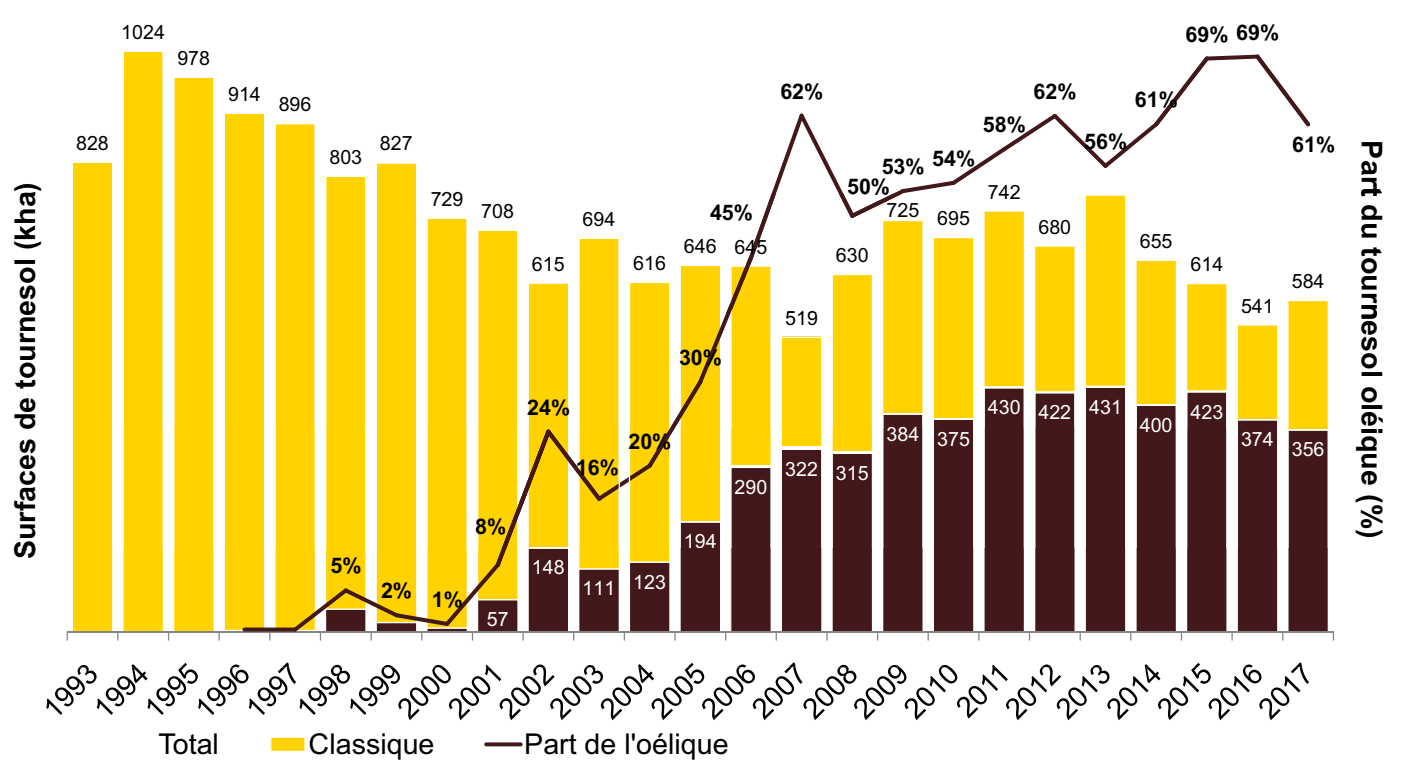

Q2 Fig. 2. Évolution historique des surfaces de tournesol classique et tournesol oléique en France. Source: Sofiprotéol (2017) d'après UFS.

\subsection{De l'utilisation du HOSO pur au «blend»}

Les années 2010 ont vu l'émergence d'une nouvelle tendance : le recours des industries agroalimentaires et de la restauration à des mélanges de plusieurs huiles dits «blends». L'HOSO est l'huile avec la plus forte teneur en acide oléique mais elle est également plus chère. Le recours au blend permet de réduire le coût d'achat des matières premières tout en optimisant les propriétés techniques, voire en apportant des fonctionnalités supplémentaires.

Deux catégories d'utilisateurs finaux de l'industrie agroalimentaire et de la restauration se distinguent: d'une part, les leaders du marché ont de plus en plus recours aux blends. Ils achètent les huiles séparément et font leurs propres mélanges pour optimiser la composition en acides gras, les propriétés techniques et les prix des huiles. D'autre part, les plus petits acteurs continuent aujourd'hui d'acheter essentiellement de l'HOSO pur mais ont de plus en plus recours aux blends pour réduire leurs coûts, qu'ils achètent déjà prêts auprès de leurs fournisseurs. L'ensemble des utilisateurs finaux expriment cependant leur besoin en conseil et expertise de leurs fournisseurs.

\subsection{De multiples drivers de valeur viennent contrebalancer les facteurs de volatilité du prix du tournesol oléique}

De nombreux paramètres peu prévisibles peuvent influer à la hausse ou à la baisse sur le prix du tournesol oléique :

- mauvaise récolte de tournesol oléique;

- surcoûts logistiques;

- des quantités significatives qui arriveraient sur le marché (nouvelles origines)...;

- ...ou une forte hausse de la production dans les principaux pays producteurs ;

- des utilisateurs finaux prêts à payer une forte prime sur des volumes importants ;
- une situation contrainte («short») d'un industriel;

- des positions spéculatives prises par des traders.

À l'inverse, pour le client final, un premium de prix se justifie par d'autres critères qui font la valeur de ce produit:

- réponse à des besoins clients ou usages complexes ;

- ajout de nouvelles fonctionnalités à l'huile, comme des antioxydants, des anti-moussants, etc.;

- qualité du fournisseur/image de marque et fiabilité pour les mélanges : garantie pour la sécurité alimentaire;

- origine de l'huile (qualité, traçabilité...);

- flexibilité des volumes d'approvisionnement, conditionnement, transport...

Ces drivers de la valeur qui s'appuient sur l'usage final de l'huile sont un levier majeur à exploiter pour atténuer l'instabilité du marché.

\section{La filière érucique pour une diversité de débouchés en oléochimie}

\subsection{Le colza érucique, un colza ancestral riche en acides gras à longues chaînes}

Le colza érucique est le seul oléagineux sous nos latitudes à potentiel de rendement intéressant contenant des acides gras longs, notamment en acide érucique, chaîne de 22 atomes de carbone. C'était le colza historiquement cultivé mais sa forte teneur en acide érucique ( $50 \%$ dans sa fraction lipidique) a été, dans les années 1970, jugée toxique pour l'alimentation humaine. Des variétés de colza 00 ( $0,4 \%$ d'acide érucique) ont alors été développées pour adresser les marchés alimentaires en Europe et en Amérique du Nord (Tab. 1). Si les fondements scientifiques sur la toxicité de l'acide érucique font encore aujourd'hui l'objet de controverses en Occident, l'Inde et le Pakistan sont de grands consommateurs d'huile de moutarde, 
Tableau 1. Composition de la fraction lipidique du colza érucique et du colza 00 .

\begin{tabular}{lcc}
\hline Acides gras & Colza érucique & Colza 00 \\
\hline Palmitique C16:0 & 2,5 & 3,5 \\
Stéarique C18:0 & 1 & 1,5 \\
Oléique C18:1 & 14 & 61,5 \\
Linoléique C18 :2 & 12,5 & 19,5 \\
Linolénique C18:3 & 8 & 10 \\
Érucique C22:1 & 50 & 0,4 \\
Divers & 11,5 & 3,6 \\
\hline
\end{tabular}

qui contient pourtant plus de $40 \%$ d'acide érucique (ce qui apporterait un petit goût piquant très apprécié dans les cuisines locales).

Aujourd'hui, la production de colza française est quasi exclusivement 00 mais une filière de niche s'est développée en érucique à destination des marchés à haute valeur ajoutée pour l'oléochimie. Les débouchés ont multiples:

- fabrication d'érucamide:

- en additif pour les matières plastiques (effet antisticking qui empêche les films plastiques de coller ensemble),

- en tensioactif dans les formulations pour améliorer la récupération assistée du pétrole brut,

- en tensioactif dans des formulations cosmétiques;

- transformation en alcool béhénique pour abaisser le point d'écoulement des formulations grasses;

- accès à des molécules en C13 et C9, notamment l'acide brassilique et l'acide pélargonique pour des applications en plastifiants, parfums ou encore production de nylon.

\subsection{Un marché à valeur ajoutée pour les producteurs}

La filière érucique française a été créée il y une vingtaine d'années dans le but d'offrir aux agriculteurs l'opportunité de s'engager sur une production à valeur ajoutée avec une vision long terme, en co-construction avec les clients. Cette filière s'est bâtie sur le rapprochement entre les groupes oléochimistes qui avaient besoin d'huile érucique et les coopératives qui ont mis en place des contrats avec engagements sur la durée. Pendant 15 ans, cette filière est restée confidentielle avant de prendre de l'ampleur ces dernières années - tout en restant une culture de niche.

En 2015, cette filière représentait 20 kha de cultures de colza érucique, soit $85 \mathrm{kt}$ de graines triturées dans l'usine Saipol de Dieppe pour produire $38 \mathrm{kt}$ d'huile érucique.

Pollen est aujourd'hui l'acteur majeur du colza érucique en Europe. Partenariat créé en 1991 par quatre coopératives, Pollen est devenu une SAS en 2012 regroupant Cap Seine, Sevépi, Interface Céréales et Sofiprotéol.

\subsection{Enjeux et facteurs clé de succès}

Pollen est fortement impliqué dans une démarche filière puisqu'il contractualise en amont avec les adhérents producteurs, en aval pour la commercialisation de l'huile et soutien la recherche génétique. Pour poursuivre la «success story » de cette filière, il faudra continuer à :

- adapter la quantité produite aux besoins industriels ;

- convaincre les agriculteurs de s'engager;

- mettre en place des contrats avec engagement sur la durée :

- avec les industriels : pour coller aux besoins du marché et produire au plus près la juste quantité en s'engageant par contrats sur plusieurs années,

- avec les agriculteurs: contractualiser sur plusieurs années avec engagement sur une prime moyenne minimale. À noter que cet engagement dans le temps permet de garantir la séparation entre les zones de cultures érucique et classique et d'éviter les repousses de variétés éruciques dans des parcelles cultivées en colza classique,

- développer un outil original avec tunnel de prime pour les producteurs,

- investir dans l'innovation variétale pour être compétitif par rapport au colza alimentaire.

Ce système de contractualisation sur l'ensemble de la chaîne de valeur permettra de protéger la filière d'une forte volatilité des prix et de la prime et d'éviter ce qui s'est passé sur le tournesol oléique.

\section{La filière Fleur de Colza, démarche de long terme de partenariat agricole}

La filière développée dans cette partie est un exemple de diversification dans le marché de l'huile de colza alimentaire. La marque Fleur de Colza, lancée par Lesieur en 2005, est une démarche de long terme de partenariat avec le monde agricole avec pour objectifs (i) la maîtrise des processus de production, (ii) la traçabilité, (iii) le respect de l'environnement et (iv) l'emploi local, en réponse aux évolutions de la recherche scientifique et des attentes des consommateurs.

Cette filière est entièrement contractualisée, de l'amont à l'aval, avec 1000 agriculteurs produisant les graines de colza sous cahier des charges spécifique qui sont collectées par 5 organismes stockeurs : Axéréal, Bonneval Beauce et Perche, Interface Céréales, SCAEL et Soufflet. Les graines sont triturées par Saipol dans son usine de Grand-Couronne (SeineMaritime) et l'huile est embouteillée dans l'usine Lesieur de Coudekerque (Nord). Un organisme de contrôle indépendant garantit le respect du cahier des charges Fleur de Colza sur tous les maillons de la chaîne.

Le cahier des charges Fleur de Colza est organisé en deux volets. La traçabilité, d'une part, avec des procédures visant à garantir l'origine et à préserver l'identité des lots de graines de la parcelle jusqu'à l'usine. Cela permet de valoriser dans le produit fini une origine France et même régionale pour les producteurs. D'autre part, le cahier des charges définit des conditions de production des graines afin d'obtenir une huile de qualité et produite dans le respect de l'environnement. Cela passe par l'utilisation de semences certifiées uniquement et selon la liste des variétés recommandées et interdites en fonction de leur teneur en oméga-3, ainsi que par le respect des règles de la «Charte Environnement Fleur de Colza » pour des pratiques culturales respectueuses de l'environnement: choix des variétés, date de semis, traitements phytosanitaires, 
fertilisation azotée, rotation des cultures et gestion des espaces de l'exploitation agricole. Ce cahier des charges évolue d'année en année afin d'améliorer constamment l'efficacité environnementale de la filière.

Pour animer cette démarche de progrès, Lesieur a développé un partenariat pluriannuel avec Terres Inoviacentre technique interprofessionnel des oléagineux, des protéagineux et du chanvre. Grâce à son expertise, Terres Inovia conçoit des formulaires d'enquête sur les pratiques culturales des agriculteurs sous cahier des charges Fleur de Colza, recueille les résultats et analyses les bases de données pour calculer des indicateurs environnementaux sur la consommation des ressources, la qualité de l'eau, la fertilité des sols ou encore la biodiversité. Ces indicateurs permettent ensuite d'évaluer la performance de la démarche Fleur de Colza, d'identifier les facteurs d'amélioration et de construire un plan d'action pour communiquer sur la filière et faire évoluer le cahier des charges.

Après plus de dix d'existence, la filière Fleur de Colza se différencie des huiles de colza sous marque distributeur par sa double promesse de traçabilité et de richesse en oméga-3. Son organisation en filière permet de valoriser les producteurs tout en contribuant à l'amélioration des pratiques culturales pour améliorer l'empreinte environnementale.

\section{La filière bio, un marché jeune, dynamique, en cours de structuration}

\subsection{Une forte dynamique récente sur les grandes cultures en bio, en retard sur les autres filières}

Le marché français du bio - tous produits confondus - est en plein développement, avec $+21 \%$ de croissance entre 2015 et 2016 pour atteindre un chiffre d'affaires total de plus de 7,1 Mds $€$ (Agence Bio, 2017). Ce marché a doublé de taille depuis 2010, porté par les nouvelles attentes sociétales et environnementales des consommateurs vis-à-vis de leur alimentation, de leur capital santé, etc. Le potentiel de développement est encore grand puisque le bio ne représente encore que 3,5\% du marché de la consommation alimentaire à domicile des ménages. Cette croissance récente et à venir implique une nécessaire adaptation de l'offre pour répondre aux attentes du consommateur en termes de qualité, de proximité - «locavore »- et de traçabilité.

La production agricole s'adapte à la demande mais elle reste encore insuffisante, notamment en grandes cultures. En effet, le taux de pénétration du bio diffère beaucoup selon les filières agricoles. En 2016, 1,54 millions d'hectares étaient certifiées bio ou en conversion en France, en hausse de $17 \%$ par rapport à 2015, atteignant $5,7 \%$ des surfaces totales. Les surfaces fourragères représentent l'essentiel de ces surfaces avec près d'un million d'hectares. Si la pénétration du bio dans les surfaces de fourrage, vigne et maraichage varie entre $8 \%$ et $9,4 \%$, la part du bio dans les grandes cultures n'est que de $3 \%$ pour les grandes cultures. En 2016, seulement $357 \mathrm{kha}$ de grandes cultures étaient en bio, dont 139 kha en conversion. Ce sont néanmoins les filières qui ont connu les plus forts taux de croissance des surfaces de bio entre 2015 et 2016, grâce aux aides à la conversion attribuées aux producteurs et aux difficultés économiques touchant l'agriculture conventionelle.
Tableau 2. Surfaces en bio en 2016 pour les filières végétales.

\begin{tabular}{lccll}
\hline Filières végétales & $\begin{array}{l}\text { Surfaces } \\
\text { certifiées } \\
2016(\mathrm{kha})\end{array}$ & $\begin{array}{l}\text { Surfaces en } \\
\text { conversion }\end{array}$ & $\begin{array}{l}\text { Surfaces } \\
\text { totales }\end{array}$ & $\begin{array}{l}\text { Part du } \\
\text { bio 2016 } \\
(\%)\end{array}$ \\
\hline Fourrage & 690 & 306 & 996 & 8,0 \\
Maraichage & 44 & 10 & 54 & 9,4 \\
Vigne & 59 & 12 & 71 & 9,0 \\
Grandes cultures & 218 & 139 & 357 & 3,0 \\
$\quad$ dont céréales & 165 & 102 & 267 & 2,8 \\
dont oléagineux & 29 & 24 & 54 & 2,4 \\
Autres & 44 & 16 & 60 & - \\
Total & 1055 & 493 & 1538 & 5,7 \\
\hline
\end{tabular}

Source: d'après Agence Bio (2017).

La production d'oléagineux bio est encore plus limitée, avec 60 kha en 2016 dont 16 kha en conversion (Tab. 2).

\subsection{Encore peu d'oléagineux bio par rapport aux besoins des triturateurs}

La moitié des surfaces nationales d'oléagineux bio sont du soja, avec un forte progression ces dernières années (Terres Univia, 2017). Le soja bio représente $18 \%$ des surfaces françaises de soja, dépassant depuis 2014 le tournesol bio, porté par les hausses des demandes en fèves pour l'alimentation humaine (lait de soja, steak végétal, etc.) en tourteaux pour l'alimentation animale (pour la production de viandes, de lait et d'œufs bio). Le tournesol bio représente l'essentiel des autres surfaces d'oléagineux bio mais stagne depuis quelques années. La culture du colza bio a démarré très récemment mais reste encore confidentielle (Fig. 3). Il est en effet difficile techniquement de produire du colza bio tout en restant compétitif.

Malgré la croissance récente des surfaces, la France demeure importatrice d'oléagineux bio pour satisfaire la demande. Sur la campagne 2015/16, $15 \%$ du soja bio consommé était importé. Les deux tiers des utilisations sont à destination du marché de l'alimentation humaine et le tiers restant pour la production de tourteaux bio pour l'alimentation animale. Ces deux marchés sont en forte croissance mais la hausse de la production de soja bio français a permis de réduire la dépendance aux importations. Près de $40 \%$ des graines de tournesol bio consommées étaient importées sur la campagne 2015/16, en hausse du fait d'un repli de la collecte française. La production domestique de graines de colza bio est encore très faible, si bien que les industriels satisfont leur demande par l'importation (77\% de la demande en 2015/16). En plus de l'importation de ces trois graines bio, il est important de noter que les fabricants d'aliment du bétail ont recours à l'importation de tourteaux bio de soja et de tournesol: environ 40 milles tonnes de ces tourteaux auraient été importés sur la campagne 2015/16.

\subsection{Un marché en cours de structuration pour l'amont agricole}

L'amont agricole doit s'adapter pour tirer profit de ce marché en croissance, mais les freins subsistent pour pouvoir 


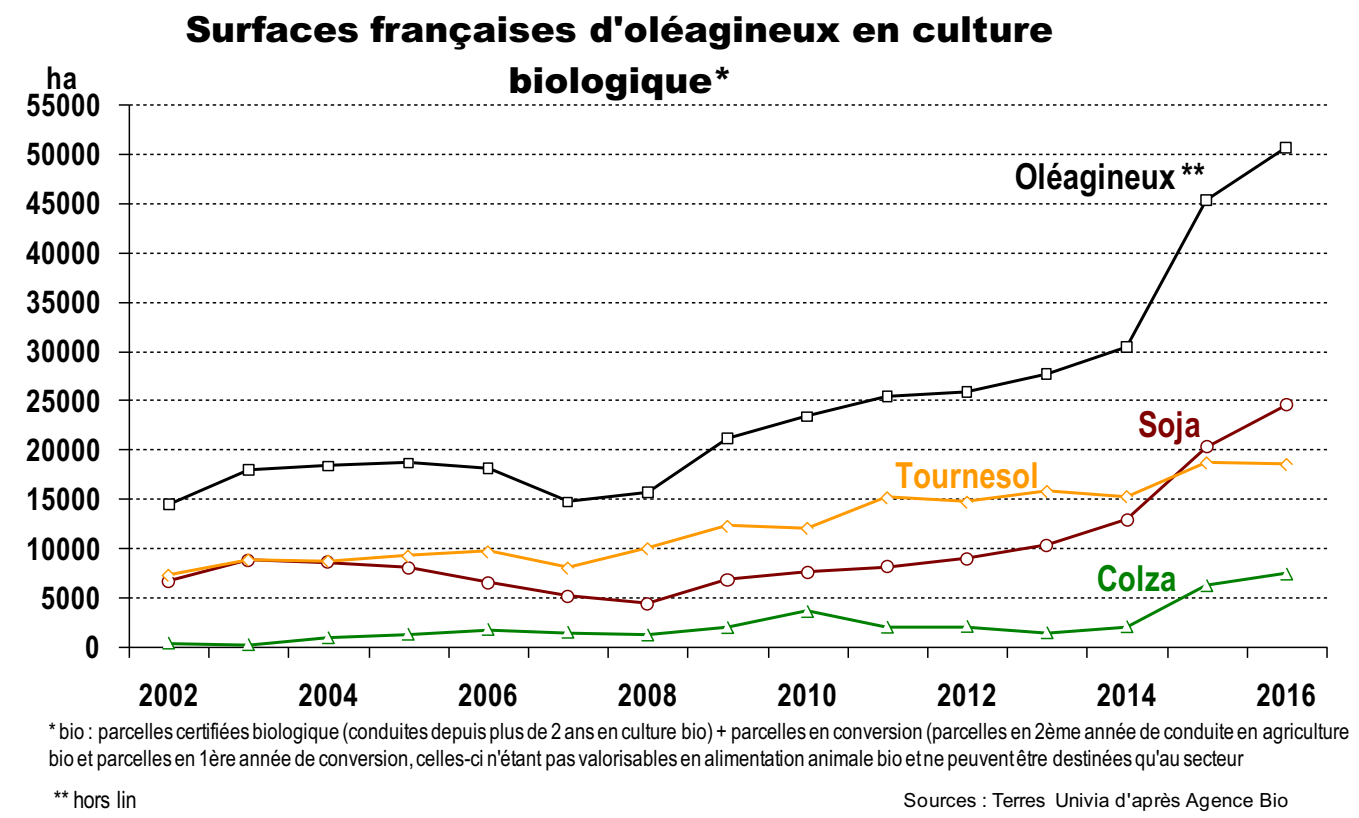

Fig. 3. Évolution historique des surfaces d'oléagineux bio en France. Source: Terres Univia (2017) d'après Agence Bio (2017).

répondre à la demande des consommateurs et trouver des relais de croissance et de compétitivité :

- moindre performances agronomiques en agriculture biologique;

- grande variabilité interannuelles des productions biologiques ;

- manque de visibilité sur les amortissements des installations de stockage spécifiques;

- incertitudes sur l'évolutions des prix agricoles bio à moyen terme ;

- manque de solutions alternatives à la lutte phytosanitaire en France, sous contrainte du cahier des charges pour l'agriculture biologique ;

- interprétation différente du cahier des charges pour l'agriculture biologique selon les États Membres de l'Union Européenne et concurrence avec les produits de l'agriculture biologique hors Union Européenne dont les cahiers des charges diffèrent.

\section{Conclusion}

Les quatre exemples de filières de spécialité des productions oléagineuses développées dans cet article sont des exemples de structuration des filières agricoles françaises pour répondre à la demande du marché de diversification des productions et sécuriser des nouveaux débouchés pour les producteurs tout en maintenant de la valeur ajoutée au niveau de l'amont. Toutefois, ces spécialités ne peuvent être valorisées sans un accompagnement de l'ensemble des acteurs de ces filières pour:

- valoriser l'origine française par la traçabilité;
- former les producteurs aux techniques culturales spécifiques à ces productions de spécialité et développer l'offre en conseil ;

- optimiser la chaîne logistique et le stockage au niveau des organismes stockeurs;

- fédérer l'ensemble des membres de la filière, notamment via la contractualisation pluriannuelle avec l'amont et l'aval ;

- innover techniquement via la sélection variétale ou encore le développement du bio-contrôle.

\section{Références}

Agence Bio. 2017. Les chiffres de la bio en France. http:/www. agencebio.org/la-bio-en-france (dernière consult. : 28/02/2018).

Agreste, Ministère de l'Agriculture et de l'alimentation. 2017. Grandes cultures et fourrages. http://agreste.agriculture.gouv.fr/ conjoncture/grandes-cultures-et-fourrages/ (dernière consult. : 28/02/2018).

Duflot V. 2017. Produits de niches et cultures de diversification : à la recherche de valeur ajoutée. OCL 24(5): D501.

Sofiprotéol. 2017. Les productions françaises d'oléagineux de spécialité : diversité \& segmentation. http://www.franceagri mer.fr/content/download/50552/485726/file/05\%20-\%20Pr\% C3\%A9sentation\%20D\%C3\%A9bouch\%C3\%A9s\%20O1\%C3\% A9agineux $\% 20 \mathrm{de} \% 20 \mathrm{sp} \% \mathrm{C} 3 \% \mathrm{~A} 9$ cialit $\% \mathrm{C} 3 \% \mathrm{~A} 9$.pdf (dernière consult. : $28 / 02 / 2018$ ).

Terres Univia, l'interprofession des huiles et protéines végétales. 2017. Historique. http://www.terresunivia.fr/decouvrir-terres-uni via/l-interprofession/historique (dernière consult. : 28/02/2018).

Citation de l'article : Tonin P. 2018. Les productions françaises d'oléagineux de spécialité : des démarches en filière pour créer de la valeur dans nos territoires. OCL 25(2): D203. 